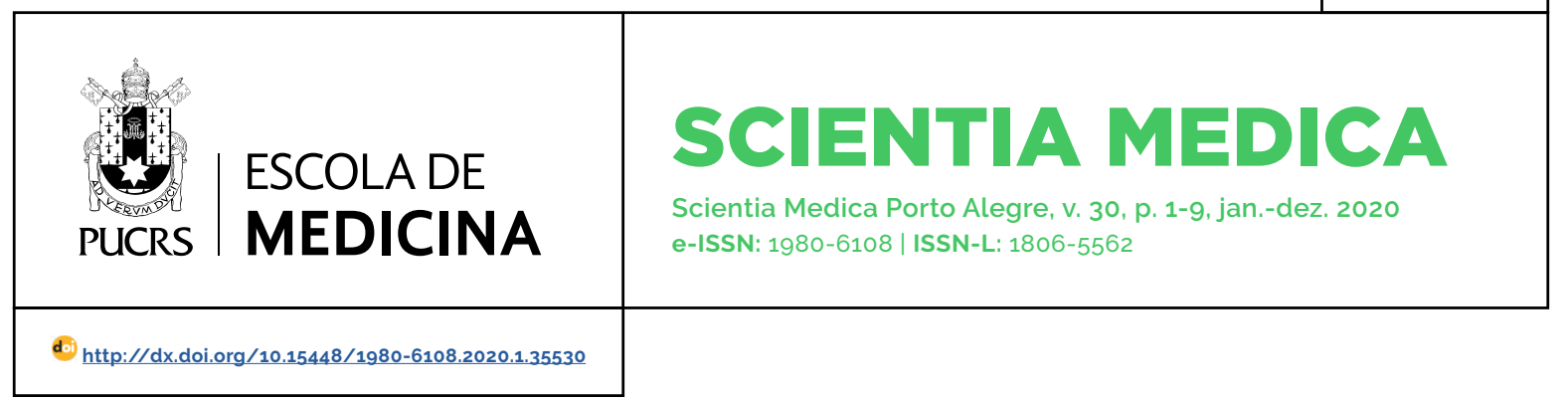

ARTIGO ORIGINAL

\title{
Fatores associados aos tipos de dificuldades alimentares em crianças entre o e 10 anos de idade: um estudo retrospectivo em um centro de referência brasileiro
}

\author{
Factors associated with the different types of feeding difficulties in children aged o to \\ 10 years: a retrospective study in a Brazilian reference center
}

\section{Arissa Matsuyama Okuizumi $^{1}$ \\ orcid.org/0000-0001-7925-1993 arissa.okuizumi@hotmail.com}

\section{Juliana Masami Morimoto 2}

orcid.org/0000-0003-3394-1723 juliana.morimoto@mackenzie.br

Luana Romão Nogueira ${ }^{1}$ orcid.org/0000-0003-1125-5765 luanaromaon@hotmail.com

\section{Priscila Maximino ${ }^{1}$} orcid.org/0000-0003-2877-0712 primaximino@gmail.com

\author{
Mauro Fisberg ${ }^{1,3}$ \\ orcid.org/0000-0003-2992-3215 \\ mauro.fisberg@gmail.com
}

Recebido em: 4 set. 2019 Aprovado em: 16 nov. 2019. Publicado em: 15 jul. 2020.

\section{(C) (i)}

Artigo está licenciado sob forma de uma licença Creative Commons Atribuição 4.0 Internacional.

\section{Resumo}

Objetivo: identificar fatores associados à prevalência dos diferentes tipos de dificuldades alimentares em crianças com idade entre 0 e 10 anos.

Métodos: trata-se de um estudo observacional retrospectivo com dados de crianças de 0 a 10 anos diagnosticadas com alguma dificuldade alimentar. atendidas em um centro de referência localizado no municipio de São Paulo, SP, Brasil. A presença da dificuldade alimentar foi analisada de acordo com o diagnóstico multidisciplinar, o aparecimento da queixa, os dados sobre a gestação e a amamentação, o estilo parental, a presença de causas orgânicas e as complicações associadas. Os dados foram tabulados no programa Microsoft Excel e as análises estatísticas foram realizadas no programa IBM SPSS Statistics 21 através de frequências absolutas e relativas, média, desvio padrão, teste do qui-quadrado e Kruskall-Wallis com o teste de post-hoc de Dunn.

Resultados: foram avaliados 166 pacientes com um predomínio do sexo masculino, em idade pré-escolar e nascidos a termo. O diagnóstico mais prevalente foi de seletividade alimentar, sem doenças orgânicas ou reações associadas. Os diagnósticos de dificuldade alimentar apresentaram associação estatisticamente significativa com idade, idade gestacional de nascimento, idade e fase da alimentação de aparecimento da queixa.

Conclusões: os fatores que apresentaram associação com os diferentes tipos de dificuldade alimentar foram a idade, a idade gestacional de nascimento e a idade e fase da alimentação de aparecimento da queixa. O presente estudo permite iniciar um direcionamento mais especifico de ações de orientação e de prevenção de dificuldade alimentar. Dessa forma, destaca-se a importância da realização de estudos desse cunho.

Palavras-chave: criança, alimentação infantil, fatores de risco.

\section{Abstract}

Aims: To identify factors associated with the prevalence of different types of feeding difficulties in children aged 0 to 10 years.

Methods: This is a retrospective observational study with data from children aged 0-10 years diagnosed with some feeding difficulty, attended at a reference center located in the city of São Paulo, Brazil. The presence of feeding difficulty was analyzed according to the multidisciplinary diagnosis, appearing of the complaint, information about pregnancy and breastfeeding, parenting style, presence of organic causes and associated complications. Data were tabulated using Microsoft Excel software and statistical analyzes were performed using IBM SPSS Statistics 21 using absolute and relative frequencies, mean, standard deviation, chi-square test and Kruskall-Wallis test associated with Dunn post-hoc test.

Results: 166 patients were analysed, with male, preschool age and full-term

\footnotetext{
Instituto Pesquisa e Ensino em Saude Infantil (PENSI), São Paulo, São Paulo, Brasil.

Universidade Presbiteriana Mackenzie, São Paulo, SP, Brasil.

Universidade Federal de São Paulo (UNIFESP), São Paulo, SP, Brasil.
} 
predominance. The most prevalent diagnosis was picky eating, with no associated organic diseases or reactions. Diagnoses of feeding difficulty were statistically significant associated with age, gestational age at birth, age and feeding phase of onset of the complaint.

Conclusions: The factors that were associated with the different types of feeding difficulty were age, gestational age at birth, age and feeding phase of emergence of the complaint. The present study allows us to initiate a more specific direction of orientation actions and prevention of feeding difficulties, thus highlighting the importance of conducting studies of this nature.

Keywords: child, child nutrition, risk factors.

ABREVIATURAS: DA, dificuldade(s) alimentar(es); AME, aleitamento materno exclusivo.

\section{Introdução}

Dificuldades alimentares (DA) consistem em empecilhos qualitativos e/ou quantitativos ao processo de alimentação $(1,2)$ que podem apresentar diversos motivos, como causas orgânicas e comportamentais (3), assim, sendo consideradas condições multifatoriais (4). Pouco apetite, recusa alimentar, desinteresse pelos alimentos, refeições muito longas e aspectos relacionados ao comportamento, como agitação, distração e negociações, são citados como alguns desses problemas alimentares $(1,5)$.

As DA geram preocupação, insatisfação e insegurança nos pais (6), visto que a alimentação tem papel primordial nos processos de crescimento e desenvolvimento infantil (7). Os possiveis déficits no desenvolvimento cognitivo, nutricional e emocional causados por DA $(8,9)$ podem perdurar mesmo na fase adulta (10). A complexidade desses casos ganha destaque na literatura, devido a fatores como a heterogeneidade e escassez de dados (4, 11), mas apesar de ser um assunto em ascendência, com aumento da produção científica, ainda necessita de maiores esclarecimentos.

Assim, torna-se relevante a realização de estudos a fim de reconhecer fatores de risco em casos confirmados de DA, possibilitando o desenvolvimento de ações de prevenção, reduzindo a ocorrência, e promovendo o crescimento e o desenvolvimento adequado da população infantil. Portanto, o objetivo do presente estudo é identificar fatores associados à prevalência dos diferentes tipos de dificuldades alimentares em crianças com idade entre o e 10 anos.

\section{Metodologia}

Trata-se de um estudo observacional retrospectivo, com dados de crianças de o a 10 anos (lactentes, préescolares e escolares) diagnosticadas com alguma DA, atendidas no Centro de Dificuldades Alimentares, um centro de referência localizado no municipio de São Paulo, SP. Foram excluidos do estudo pacientes cujo diagnóstico estava em aberto e/ou pacientes com ausência de qualquer informação necessária para essa pesquisa.

A presença da DA foi analisada de acordo com o diagnóstico multidisciplinar, a percepção do responsável pela criança quanto à DA (idade e fase da alimentação em que o comportamento foi notado), dados sobre a gestação e amamentação (idade gestacional no nascimento, se foi realizado, e periodo de aleitamento materno exclusivo), estilo parental, presença de causas orgânicas e complicações associadas.

O diagnóstico do paciente é baseado na classificação de Kerzner com adaptações (2), já que não há instrumentos validados para tal função. O centro de referência considera a classificação das DA em seletividade alimentar. apetite limitado e fobia alimentar.

O estilo parentalé definido de acordo com Hughes e colaboradores (12), cujo instrumento classifica os responsáveis em responsivos, controladores, indulgentes e não envolvidos, e relaciona essa variável ao comportamento alimentar infantil.

Os dados foram tabulados no programa Microsoft Excel e as análises estatísticas foram realizadas no programa IBM SPSS Statistics 21. As variáveis qualitativas do estudo foram demonstradas por meio de frequências absolutas e relativas, apresentadas em tabelas. Já as variáveis quantitativas, tiveram média e desvio padrão calculados.

Para analisar a associação entre as classificações da DA e as variáveis qualitativas do estudo foi utilizado o teste do qui-quadrado. Como todas as variáveis quantitativas não tiveram aderência à distribuição normal, utilizou-se o teste não paramétrico de Kruskal-Wallis para analisar 
as diferenças entre as médias dessas variáveis segundo categorias da variável diagnóstico de DA, seguido do teste post-hoc de Dunn. Em todos os testes estatísticos foi considerado nivel de significância de $5 \%$

Todos os procedimentos dessa pesquisa respeitaram as diretrizes da legislação de ética em pesquisa com seres humanos. $O$ uso de dados de prontuários foi previamente aprovado pelo Comitê de Ética em Pesquisa da Fundação José Luiz Egydio Setúbal para projeto de pesquisa anterior (registrados sob Certificado de Apresentação para Apreciação Ética 32939314.0.0000.5567), bem como o presente estudo (Certificado de Apresentação para Apreciação Ética 18189419.8.0000.5567). Todos os pacientes apresentam Termo de Consentimento Livre e Esclarecido assinado pelo responsável legal.

\section{Resultados}

A amostra foi composta por 166 pacientes, com idade média de 44,66 $\pm 31,10$ meses, com tempo médio de AME de 3,29 22,76 meses. As caracteristicas gerais da amostra estão descritas na Tabela 1.

TABELA 1 - Caracteristicas gerais de crianças com dificuldades alimentares. Instituto PENSI, Brasil, 2018

\begin{tabular}{|c|c|c|}
\hline Variáveis & $\mathrm{n}$ & $\%$ \\
\hline \multicolumn{3}{|l|}{ Sexo } \\
\hline Feminino & 58 & 34.9 \\
\hline Masculino & 108 & 65,1 \\
\hline \multicolumn{3}{|l|}{ Idade (meses) } \\
\hline ol-- 24 & 40 & 24,1 \\
\hline $24 \mid--84$ & 107 & 64.5 \\
\hline $84 \mid--120$ & 19 & 11,4 \\
\hline \multicolumn{3}{|c|}{$\begin{array}{l}\text { Idade gestacional de nascimento } \\
\text { (semanas) }\end{array}$} \\
\hline$<36$ & 21 & 12,6 \\
\hline $36-38$ & 37 & 22,3 \\
\hline $38-42$ & 108 & 65,1 \\
\hline \multicolumn{3}{|l|}{ Prática de AME } \\
\hline Sim & 125 & 75,3 \\
\hline Não & 41 & 24,7 \\
\hline
\end{tabular}

\begin{tabular}{|c|c|c|}
\hline Variáveis & $\mathrm{n}$ & $\%$ \\
\hline \multicolumn{3}{|l|}{ Tempo de AME (meses) } \\
\hline Nunca amamentou & 41 & 24,7 \\
\hline $0,1 \mid--6$ & 71 & 42,8 \\
\hline $6 \mid--12$ & 52 & 31,3 \\
\hline$\geq 12$ & 2 & 1,2 \\
\hline \multicolumn{3}{|l|}{ Diagnóstico de DA } \\
\hline Apetite limitado & 58 & 34.9 \\
\hline Fobia alimentar & 8 & 4,8 \\
\hline Seletividade alimentar & 100 & 60,3 \\
\hline \multicolumn{3}{|l|}{ Doença orgânica associada } \\
\hline Alergia & 13 & 7,8 \\
\hline Anatômica & 3 & 1,8 \\
\hline Cardiorrespiratória & 2 & 1,2 \\
\hline Relacionadas ao crescimento & 5 & 3,0 \\
\hline Gástrica & 14 & 8,5 \\
\hline Neurológica & 16 & 9,6 \\
\hline Relacionadas à prematuridade & 9 & 5,4 \\
\hline Sindrômica & 2 & 1,2 \\
\hline Sem doença orgânica associada & 106 & 63.9 \\
\hline $\begin{array}{l}\text { Mais de uma doença orgânica } \\
\text { associada }\end{array}$ & 4 & 2,4 \\
\hline \multicolumn{3}{|l|}{ Outras reações associadas } \\
\hline Agitado & 6 & 3,6 \\
\hline Choro & 17 & 10,2 \\
\hline Cuspe & 22 & 13.3 \\
\hline Náusea & 5 & 3,0 \\
\hline Sono & 1 & 0,6 \\
\hline Vômito & 38 & 22,9 \\
\hline Sem outras reações associadas & 85 & 51,2 \\
\hline Mais de uma reação associada & 8 & 4,8 \\
\hline \multicolumn{3}{|l|}{ Estilo parental } \\
\hline Controlador & 60 & 36,2 \\
\hline Indulgente & 46 & 27,7 \\
\hline Não envolvido & 16 & 9,6 \\
\hline Responsivo & 44 & 26,5 \\
\hline Total & 166 & 100 \\
\hline
\end{tabular}

AME, aleitamento materno exclusivo; DA, dificuldade alimentar.

A maioria dos pacientes apresentou início da queixa enquanto lactente (entre o e 23.9 meses) $(80,1 \%)$ e pré-escolar (entre 24 e 84,9 meses) $(19,9 \%)$ 
sendo que não houve relato de casos em que a queixa se iniciou na fase escolar (entre $85 \mathrm{e}$ 119,9 meses). Quanto à fase da alimentação do aparecimento da queixa, a prevalência foi maior na transição para a alimentação da família $(48,8 \%)$, seguido pela alimentação complementar (39,8\%) e pelo aleitamento materno $(11,4 \%)$, respectivamente.

Quando analisadas a distribuição das variáveis qualitativas segundo o diagnóstico de DA é possivel observar uma diferença estatisticamente significativa nas variáveis idade gestacional de nascimento e fase da alimentação de aparecimento da queixa (Tabela 2). Não houve associação estatisticamente significante entre os diagnósticos de DA no que diz respeito à prática de AME e ao estilo parental. O diagnóstico de DA e a idade gestacional de nascimento apresentaram relação $(p=0,01)$, sendo que a fobia alimentar foi mais prevalente em pacientes nascidos pré-termo, com idade gestacional inferior a 36 semanas. A associação entre o diagnóstico de DA e a fase da alimentação em que surgiu a queixa $(p<0,001)$ mostrou que a queixa de apetite limitado teve início com maior frequência na fase da alimentação complementar, enquanto a queixa de seletividade alimentar comumente se iniciou na fase da alimentação da família.

TABELA 2 - Distribuição das variáveis qualitativas segundo diagnóstico de dificuldade alimentar. Instituto PENSI, Brasil, 2018

\begin{tabular}{|c|c|c|c|c|c|c|c|c|c|}
\hline \multirow{3}{*}{ Variáveis } & \multicolumn{6}{|c|}{ Diagnóstico de DA } & \multirow{2}{*}{\multicolumn{2}{|c|}{ Total }} & \multirow{3}{*}{$\begin{array}{c}\text { Valor } \\
p^{*}\end{array}$} \\
\hline & \multicolumn{2}{|c|}{$\begin{array}{l}\text { Apetite } \\
\text { limitado }\end{array}$} & \multicolumn{2}{|c|}{$\begin{array}{l}\text { Fobia } \\
\text { alimentar }\end{array}$} & \multicolumn{2}{|c|}{$\begin{array}{l}\text { Seletividade } \\
\text { alimentar }\end{array}$} & & & \\
\hline & $\mathrm{n}$ & $\%$ & $\mathrm{n}$ & $\%$ & $\mathrm{n}$ & $\%$ & $\mathrm{n}$ & $\%$ & \\
\hline \multicolumn{10}{|l|}{ Sexo } \\
\hline Feminino & 27 & 46,5 & 3 & 37.5 & 28 & 28,0 & 58 & 34,9 & 0,060 \\
\hline Masculino & 31 & 53,4 & 5 & 62,5 & 72 & 72,0 & 108 & 65,1 & \\
\hline \multicolumn{10}{|c|}{ Idade gestacional de nascimento (semanas) } \\
\hline$<36$ & 9 & 15.5 & 4 & 50,0 & 8 & 8,0 & 21 & 12,6 & 0,010 \\
\hline $36-38$ & 13 & 22,4 & 2 & 25,0 & 22 & 22,0 & 37 & 22,3 & \\
\hline $38-42$ & 36 & 62,1 & 2 & 25,0 & 70 & 70,0 & 108 & 65,1 & \\
\hline \multicolumn{10}{|l|}{ Prática de AME } \\
\hline $\operatorname{sim}$ & 43 & 74,1 & 6 & 75,0 & 76 & 76,0 & 125 & 75.3 & 0,966 \\
\hline Não & 15 & 25.9 & 2 & 25,0 & 24 & 24,0 & 41 & 24.7 & \\
\hline \multicolumn{10}{|l|}{ Estilo parental } \\
\hline Controlador & 25 & 43,1 & 2 & 25,0 & 33 & 33,0 & 60 & 36,1 & 0,567 \\
\hline Indulgente & 18 & 31,0 & 3 & 37.5 & 25 & 25,0 & 46 & 27,7 & \\
\hline Não envolvido & 4 & 6.9 & 1 & 12,5 & 11 & 11,0 & 16 & 9.7 & \\
\hline Responsivo & 11 & 19 & 2 & 25,0 & 31 & 31,0 & 44 & 26,5 & \\
\hline \multicolumn{10}{|c|}{ Fase da alimentação de aparecimento da queixa } \\
\hline Aleitamento materno & 13 & 22,4 & 2 & 25,0 & 4 & 4,0 & 19 & 11,4 & $<0,001$ \\
\hline Alimentação complementar & 29 & 50,0 & 3 & 37.5 & 34 & 34,0 & 66 & 39,8 & \\
\hline Alimentação da familia & 16 & 27,6 & 3 & 37,5 & 62 & 62,0 & 81 & 48,8 & \\
\hline Total & 58 & 100 & 8 & 100 & 100 & 100 & 166 & 100 & \\
\hline
\end{tabular}

*teste do qui-quadrado.

AME, aleitamento materno exclusivo; DA, dificuldade alimentar. 
Observam-se, na Tabela 3, as médias e desvios-padrão das variáveis quantitativas segundo diagnóstico de DA. Houve associação estatisticamente significativa quando analisadas as médias de idade e idade de aparecimento da queixa entre os pacientes com diagnósticos de apetite limitado e seletividade alimentar. Houve associação entre idade e diagnóstico de DA ( $p<0,001)$, sendo que o teste post-hoc de Dunn mostrou que pacientes com diagnóstico de apetite limitado apresentaram média de idade mais baixa $(31,83 \pm 25,04$ meses) quando comparada à média de idade dos pacientes com seletividade alimentar (52,92 $\pm 32,59$ meses). Verificou-se também que a idade média de aparecimento da queixa foi menor nos casos de apetite limitado (10,09 $\pm 11,51$ meses), quando comparada à idade média de surgimento dos casos de seletividade alimentar (15,75 $\pm 11,8$ meses), com diferença estatisticamente significativa pelo teste post-hoc de Dunn ( $p<0,001)$.

TABELA 3 - Média e desvio padrão das variáveis quantitativas segundo diagnóstico de dificuldade alimentar. Instituto PENSI, Brasil, 2018

\begin{tabular}{lcccc}
\hline \multicolumn{1}{c}{ Variáveis } & $\begin{array}{c}\text { Apetite } \\
\text { limitado }\end{array}$ & Fobia alimentar & $\begin{array}{c}\text { Seletividade } \\
\text { alimentar }\end{array}$ & Valor $\mathrm{p}^{*}$ \\
\hline Idade (meses) & $31,83 \pm 25,04^{+}$ & $34,37 \pm 14,18$ & $52,92 \pm 32,5^{+}$ & $<0,001$ \\
Tempo de AME (meses) & $3,00 \pm 2,72$ & $3,00 \pm 2,51$ & $3,49 \pm 2,82$ & 0,489 \\
$\begin{array}{l}\text { Idade de aparecimento } \\
\text { da queixa (meses) }\end{array}$ & $12,67 \pm 23,71^{+}$ & $10,50 \pm 9,96$ & $15,75 \pm 11,80^{+}$ & $<0,001$ \\
\hline
\end{tabular}

"teste de Kruskall-Wallis. ${ }^{\dagger} p<0,001$ no teste post-hoc de Dunn. AME, Aleitamento Materno Exclusivo.

Quando analisada a ocorrência de doenças orgânicas associada às DAs, observa-se que a maior parte dos casos, independentemente do diagnóstico, não apresentou doença orgânica associada, sendo $56,9 \%$ dos casos de apetite limitado, 37.5\% dos casos de fobia alimentar e 70\% dos casos de seletividade alimentar.

Nos casos de DA com doenças orgânicas associadas, verificou-se uma prevalência mais acentuada de doenças gástricas quando o diagnóstico é apetite limitado (13,8\%), doenças relacionadas à prematuridade em casos de fobia alimentar (25\%) e doenças neurológicas quando o paciente é classificado como seletivo (12\%), sem diferença estatisticamente significativa $(p=0,067)$.

Não foi observada associação entre o diagnóstico de DA e reações associadas $(p=0,53)$. De forma semelhante às doenças orgânicas, $44,8 \%$ dos casos de apetite limitado e 57\% dos casos de seletividade alimentar não apresentaram reações associadas. O vômito foi a complicação mais frequentemente associada aos casos de apetite limitado, fobia alimentar e seletividade alimentar (25.9\%, 50\% e 16\%, respectivamente), seguidos por cuspe e choro.

\section{Discussão}

O estudo apresentou uma amostra com predomínio do sexo masculino, em idade préescolar e nascidos a termo. O diagnóstico mais prevalente é de seletividade alimentar, sem doenças orgânicas ou reações associadas. Os diagnósticos de dificuldade alimentar apresentaram associação estatisticamente significativa com idade, idade gestacional de nascimento, e idade e fase da alimentação de aparecimento da queixa.

Um predomínio do sexo masculino foi verificado na amostra, semelhante ao estudo realizado com crianças de 0 a 4 anos incompletos a partir do banco de dados do 2018 Feeding Infants Toddlers Study, que observou uma prevalência de DA superior no sexo masculino, porém sem significância estatística (13). O assunto, no entanto, 
é controverso, de forma que outro estudo não observa relação entre sexo e DA $(p=0,19)(14)$.

Em relação aos diagnósticos de DA, a literatura não apresenta um padrão de classificação, apesar disso, alguns estudos comprovam uma maior frequência de seletividade alimentar, seguido de baixo apetite e fobia alimentar, assim como observado nos achados do presente artigo. Estudos verificaram a prevalência de $25,4 \%$ e $15,4 \%$ de seletividade alimentar, de $11,5 \%$ e $4.5 \%$ de apetite limitado e 0,3\% e 0,2\% de fobia alimentar, respectivamente $(15,16)$.

Ademais, observa-se que a maior prevalência é de casos sem doenças orgânicas associadas e, de forma semelhante, $84,3 \%$ e 82,1\% dos casos de seletividade alimentar e fobia alimentar, respectivamente, não apresentam alergias associadas (17).

Duas coortes com crianças nascidas a termo e pré-termo tiveram seus dados utilizados para avaliação de fatores de risco para menor tendência a se alimentar e para menor variedade alimentar (18). Foi constatada a menor tendência a se alimentar associada ao sexo feminino $(p=0,025)$, bem como pontuação média na escala para seletividade alimentar maior no sexo feminino $(p=0,02)$ (19). Esses dados são divergentes aos apresentados no presente estudo, visto que o predomínio é do sexo masculino, independentemente do tipo de DA e sem significância estatística.

A associação entre a idade gestacional de nascimento e os diagnósticos de DA foi observada. De forma semelhante em estudo de coorte realizado com crianças de 2 anos de idade para avaliar a influência da prematuridade e do peso de nascimento no comportamento alimentar, a menor tendência a comer relaciona-se a crianças com idade gestacional de nascimento abaixo de 30 semanas $(p=0,001)$ e abaixo de 28 semanas $(p=0,03)$ quando comparadas a crianças nascidas a termo em análise bruta (18). Há evidências de que haja risco aumentado em crianças nascidas pré-termo para o desenvolvimento de DA em $57 \%(p=0,005)$ e seletividade alimentar em 53\% $(p=0,03)$, quando comparadas a crianças nascidas a termo (14). Em outro estudo, no entanto, não foram calculadas médias de idade gestacional e não foi encontrada diferença entre pré-termos quando analisada a associação com a presença ou ausência da seletividade alimentar (11). Pesquisa realizada em uma clínica pediátrica voltada para alimentação, nutrição e crescimento (20), encontrou que crianças nascidas pré-termo apresentam mais dificuldade demonstrada na introdução de alimentos sólidos quando comparadas às crianças nascidas a termo. Uma revisão mostra que a literatura aponta que crianças nascidas pré-termo com muito baixo peso são mais propensas a desenvolver DA, devido à frequente exposição a estímulos sensoriais nocivos, como intubação e uso de sondas para alimentação, por exemplo (21).

Com relação à fase da alimentação em que acontece o aparecimento da queixa da DA, dados apontam que o início da queixa de seletividade alimentar coincide com a introdução de sólidos (8 a 10 meses de idade) e, por vezes, com a introdução alimentar (5 a 6 meses de idade) (4).

Pacientes com diagnóstico de apetite limitado apresentaram idade média inferior quando comparado à idade média de pacientes com diagnóstico de seletividade alimentar. A faixa etária predominante em crianças com DA é a de préescolares, informação convergente com a literatura, que se refere à seletividade alimentar como queixa frequente na fase pré-escolar, mas característica de crianças que começam a andar (4).

As idades médias encontradas na literatura são superiores à encontrada no presente estudo, sendo de 3,8 $\pm 0,7$ anos (45,6 \pm 8,4 meses) entre crianças com seletividade alimentar (22), e de $11,1 \pm 0,8$ anos $(133,2 \pm 9,6$ meses) tanto em crianças com seletividade alimentar, quanto em crianças com fobia alimentar, sem associações estatisticamente significativas em ambos os casos ( $p=0,06$ e $p=0,84$, respectivamente) (17). A idade e a prevalência de seletividade alimentar são proporcionais, apresentando significância estatística (13), dado apresentado também em estudo baseado no 2002 Feeding Infants Toddlers Study (23). Um estudo realizado com préescolares entre 1 e 6 anos de idade, em que um dos objetivos foi examinar as associações entre 
os comportamentos alimentares e a idade, sexo e peso relativo da criança, além do peso dos pais, apontou associação estatisticamente significativa entre maior faixa etária e maior pontuação na escala de seletividade alimentar $(p=0,01)$ (24).

Não foi encontrada associação estatisticamente significativa entre o tempo de AME e a ocorrência de DA. De forma semelhante, o mesmo ocorreu em estudo realizado em Natal, RN, na região Nordeste do Brasil: não observou relação entre o tempo de aleitamento materno e a presença de DA (15). No entanto, artigos mostram a relação entre o comportamento seletivo e o aleitamento materno exclusivo, sendo que evidências demonstram que indivíduos que realizaram AME até os 4 a 5 meses apresentaram menor risco quando comparados aos que realizaram AME por o a 1 mês (25) e maior prevalência de seletividade alimentar em individuos cujo AME durou menos de 6 meses (26).

Assim como o presente estudo, não há associação entre a seletividade alimentar e as crianças portadoras de alergia e problemas médicos de longo prazo (27). Em estudo com amostra composta por crianças de 8 a 30 meses de uma clínica especializada em alergias, por sua vez, evidenciou-se uma maior pontuação na escala para seletividade alimentar em pacientes com exclusão do leite de vaca da dieta $(p<0,01)$, alteração associada à alergia à proteina do leite de vaca (28). Contudo, colocações relacionadas à DA apontam que condições que causam dores no ato de se alimentar podem estar relacionadas ao apetite limitado, como alergias e intolerâncias alimentares, doença do refluxo gastroesofágico, apesar de raramente ser a causa do problema. A seletividade alimentar pode ser consequente de condições que levem ao atraso no desenvolvimento neurológico ou de motricidade oral, além de casos de hiper ou hipossensibilidade. Qualquer condição orgânica que resulte em dor significativa pode causar fobia alimentar, por exemplo crianças alimentadas por via enteral, odinofagia causada por esofagite (1).

A prevalência de casos de DA com reações associadas não é predominante. Diferindo do exposto acima, a associação positiva entre a neofobia e a recusa alimentar com sintomas como diarreia, constipação e vômito é citada em estudo prévio $(p<0,01)(29)$.

O presente estudo apresenta limitações, como o caráter observacional do estudo. Entretanto, os achados contribuíram para uma maior elucidação do tema, pouco encontrado na literatura, especialmente, nacional. Ademais, é importante ressaltar que o exposto permite iniciar um melhor direcionamento do tratamento e da prevenção das DA, visto a sua realização em um centro de referência com uma abordagem multidisciplinar.

Há um crescimento do tema na literatura, no entanto, é necessário ressaltar a escassez de estudos específicos sobre diagnósticos de DA, dessa forma, destaca-se a importância da realização de estudos desse cunho.

A idade média de diagnóstico da DA foi menor quando o diagnóstico foi de apetite limitado em relação à seletividade alimentar. O aparecimento da queixa de DA se dá predominantemente em lactentes (o a 23.9 meses) e na fase da alimentação da família. Observa-se que o diagnóstico de DA apresenta associação estatisticamente significativa com as variáveis idade, idade gestacional de nascimento e idade e fase da alimentação do aparecimento da queixa. O presente estudo permite iniciar um direcionamento mais específico de ações de orientação e prevenção de DA.

\section{Notas}

Parte desse trabalho foi apresentado no $15^{\circ}$ Congresso Brasileiro da Sociedade Brasileira de Alimentação e Nutrição (SBAN), São Paulo/SP, em agosto de 2019.

\section{Agradecimentos}

Ao Instituto Pesquisa e Ensino em Saúde Infantil (Instituto PENSI) pelo apoio ao Centro de Nutrologia e Dificuldades Alimentares.

\section{Apoio financeiro}

Este estudo não recebeu apoio financeiro de fontes externas. 


\section{Declaração de conflito de interesses}

Os autores declaram não haver conflitos de interesses relevantes ao conteúdo deste estudo.

\section{Contribuições dos autores}

Todos os autores fizeram contribuições substanciais para concepção, ou delineamento, ou aquisição, ou análise ou interpretação de dados; e redação do trabalho ou revisão crítica; e aprovação final da versão para publicação.

\section{Disponibilidade dos dados e responsabilidade pelos resultados}

Todos os autores declaram ter tido total acesso aos dados obtidos e assumem completa responsabilidade pela integridade destes resultados.

\section{Referências}

1. Kerzner B, Milano K, MacLean WC, Berall G, Stuart S, Chatoor I. A Practical Approach to Classifying and Managing Feeding Difficulties. Pediatrics [Internet]. 2015 [cited 2020 Mar 1];135(2): 344-53. https://dx.doi. org/10.1542/peds.2014-1630

2. Kerzner B. Clinical Investigation of Feeding Difficulties in Young Children: A Practical Approach. Clin Peditr (Phila) [Internet]. 2009[cited 2020 Mar 1]; 48: 9605. https://dx.doi.org/10.1177/0009922809336074

3. Kachani AT, Abreu CLM, Lisboa SBH, Fisberg M. Seletividade alimentar da criança. Pediatria [Internet]. 2005;27(1): 48-60.

4. Müller PW. Salazar V. Donelli TMS. Dificuldades Alimentares na Primeira Infância: Uma Revisão Sistemática. Estud Pesqui Psicol [Internet]. 2017;17(2): 635-52.

5. Sampaio ABM, Nogueira TL, Grigolon RB, Roma AM, Pereira LE, Dunker KLL. Seletividade Alimentar: uma abordagem nutricional. J Bras Psiquiatr [Internet]. 2013;62(2):164-70. http://dx.doi.org/10.1590/ $\underline{\text { S0047-20852013000200011 }}$

6. Maximino P, Machado RHV, Junqueira P, Ciari M, Tosatti AM, Ramos CC, Fisberg M. Como acompanhar a criança com dificuldade alimentar em escopo multidisciplinar? Protocolo de atendimento multiprofissional na infância e adolescência - estudo piloto J Hum Growth Dev [Internet]. 2016 [cited 2020 Mar 1];26(3): 331-40. http://dx.doi.org/10.7322/jhgd.122816

7. Fisberg M, Tosatti AM, Abreu CL. A criança que não come - abordagem pediátrico-comportamental. Blucher Medical Proceedings. $2^{\circ}$ Congresso Internacional Sabará de Especialidades Pediátricas. 2014 [cited 2020 Mar 1];1(4). https://dx.doi.org/10.5151/ medpro-2cisep-019
8. Xue Y, Lee E, Ning K, Zheng Y, Ma D, Gao H, Yang B, Bai Y, Wang P, Zhang Y. Prevalence of picky eating behavior in Chinese school-age children and associations with anthropometric parameters and intelligence quotient. A cross-sectional study. Appetite [Internet]. 2015 [cited 2020 Mar 1]; 91: 248-55. https:// dx.doi.org/10.1016/j.appet.2015.04.065

9. Jansen PW, Roza SJ, Jaddoe VWV, Mackenbach JD, Raat H, Hofman A, Verhulst FC, Tiemeier H. Children's eating behavior, feeding practices of parents and eight problems in early childhood: results from the population-based Generation R Study. Int J Behav Nutr Phys [Internet]. 2012 [cited 2020 Mar 1]; 9(130). https://dx.doi.org/10.1186/1479-5868-9-130

10. Ostberg M, Hagelin E. Feeding and sleeping pro lems in infancy - a follow up at early school age. Child Care Health Dev [Internet]. 2011 [cited 2020 Mar 2]; 37(1): 11-25. https://dx.doi.org/10.1111/j. 1365-2214.2010.01141.x

11. Taylor CM, Wernimont SM, Northstone K, Emmett PM. Picky/fussy eating in children: Review of definitions, assessment, prevalence and dietary intakes. Appetite [Internet]. 2015 [cited 2020 Mar 2]; 95: 34959. https://dx.doi.org/10.1016/j.appet.2015.07.026

12. Hughes SO, Power TG, Fisher JO, Mueller S, Nicklas TA. Revisiting a neglected construct: parenting styles in a child-feeding context. Appetite [Internet]. 2005 [cited 2020 Mar 2]; 44(1):83-92. https://dx.doi. org/10.1016/j.appet.2004.08.007

13. Horst K, Deming DM, Lesniauskas R, Carr BT, Reidy KC. Picky eating: Associations with child eating characteristics and food intake. Appetite [Internet] 2016 [cited 2020 Mar 2]; 103:286-93. https://dx.doi org/10.1016/j.appet.2016.04.027

14. Johnson S, Matthews R, Draper ES, Field DJ, Manktelow BN, Marlow N, Smith LK, Boyle EM. Eating difficulties in children born late and moderately preterm at $2 y$ of age: a prospective population-based cohort study. Am J Clin Nutr [Internet]. 2016 [cited 2020 Mar 2]; 103:406-14. https://dx.doi. org/10.3945/ajcn.115.121061

15. Maranhão HS, Aguiar RC, Lira DTJ, Sales MUF, Nóbrega NAN. Dificuldades alimentares em pré-escolares, práticas alimentares, pregressas e estado nutricional. Rev Paul Pediatr [Internet]. 2018 [cited 2020 Mar 2]; 36(1):45-54. http://dx.doi.org/10.1590/19840462/:2018:36:1:00004

16. Benjasuwantep B, Chaithirayanon S, Eiamudomkan $M$. Feeding problems in health young children: prevalence, related factors and feeding practices. Pediatric Reports [Internet]. 2005 [cited 2020 Mar 2]; 5(10): 38-42. https://dx.doi.org/10.4081\%2Fpr.2013.e10

17. Viljakainen HT, Figueiredo RAO, Rounge TB, Weiderpass E. Picky eating - A risk factor for underweight in Finnish preadolescents. Appetite [Internet]. 2019 [cited 2020 Mar 2]; 133: 107-14. https://dx.doi. org/10.1016/j.appet.2018.10.025 
18. Migraine A, Nicklaus S, Parnet P, Lange C, Monnery-Patris S, Des Robert C, Darmaun A, Flamant C, Amarger $\mathrm{V}$, Rozé JC. Effect of preterm birth and birth weight on eating behavior at 2 y of age. Am J Clin Nutr [Internet]. 2013 [cited 2020 Mar 2]; 97:1270-7. https://dx.doi.org/10.3945/ajcn.112.051151

19. Cao YT, Svensson V, Marcus C, Zhang J, Zhang JD, Sobko T. Eating behavior patterns in Chinese children aged 12-18 months ans associations with relative weight - factorial validation of the Children's Eating Behavior Questionnaire. Int J Behav Nutr Phys Act [Internet]. 2012[cited 2020 Mar 5]; 9(5). https://doi. org/10.1186/1479-5868-9-5

20. Burklow KA, McGrath AM, Valerius KS, Rudolph C. Relationship Between Feeding Difficulties, Medical Complexity, and Gestacional Age. Nutrition in Clinical Practice [Internet]. 2002 [cited 2020 Mar 5];16(6): 3738. https://doi.org/10.1177/0115426502017006373

21. Pagliaro CL, Bühler KEB, Ibidi SM, Limongi SCO. Dificuldades de transição alimentar em crianças prematuras: revisão crítica de literatura. J Pediatr [Internet]. 2016 [cited 2020 Mar 5];92(1): 7-14. http:// dx.doi.org/10.1016/j.jped.2015.05.004

22. Volger S, Xiaoyang S, Tong LM, Zhao D, Fan T, Zhang F, Ge J, Ho WM, Hays NP, Yao M. Nutrient intake and dietary patterns in children 2.5-5 years of age with picky eating behaviours and low weight-for-height. Asia Pac J Clin Nutr [Internet]. 2017 [cited 2020 Mar 5];26(1): 104-9. https://dx.doi.org/10.6133/apjcn.102015.02

23. Carruth BR, Ziegler PJ, Gordon A, Barr SI. Prevalence of picky eaters among infants and toddlers and their caregivers' decisions about offering a new food. J Am Diet Assoc [Internet]. 2004;104(1): 57-64.

24. Svensson V, Lundborg L, Cao Y, Nowicka P, Marcus $C$, Sobko T. Obesity related eating behaviour patterns in Swedish preschool children and association with age, gender, relative weight and parental weight - factorial validation of the Children's Eating Behavior Questionnaire. Int J Behav Nutr Phys Act [Internet]. 2011 [cited 2020 Mar 5];8(134). https://doi. org/10.1186/1479-5868-8-134

25. Specht IO, Rohde JF, Olsen NJ, Heitmann BL. Duration of exclusive breastfeeding may be related to eating behavior and dietary intake in obesity prone normal weight young children. PLoS ONE [Internet]. 2018 [cited 2020 Mar 5];13(7). https://doi.org/10.1371/ journal.pone.0200388

26. Galloway AT, Lee Y, Birch LL. Predictors and consequences of food neophobia and pickiness in young girls. J Am Diet Assoc [Internet]. 2003:103(6):692-8.

27. Hendricks K, Briefel R, Novak T, Ziegler P. Maternal and child characteristics associated with infant and toddler feeding practices. J Am Diet Assoc [Internet]. 2006 [cited 2020 Mar 5];106(1): 135-48.
28. Maslin K, Dean T, Arshad SH, Venter C. Fussy eating and feeding difficulties in infants and toddlers consuming cows' milk exclusion diet. Pediatr Allergy Immunol [Internet] 2015 [cited 2020 Mar 5];26(6): 5038. https://dx.doi.org/10.1111/pai.12427

29. Johnson S, Harris G. A preliminary study of the predictors of feeding problems in late infancy. J Reprod Infant Psychol [Internet] 2004 [cited 2020 Mar 5]; 22: 1838. https://doi.org/10.1080/02646830410001723760

\section{Endereço para correspondência}

Arissa Matsuyama Okuizumi

Centro de Nutrologia e Dificuldades Alimentares, Instituto PENSI

Av. Angélica, 2.071, 2. ${ }^{\circ}$ andar.

Bairro Higienópolis, 01227-200

São Paulo, SP, Brasil 